University of Wollongong

Research Online

Faculty of Engineering - Papers (Archive)

Faculty of Engineering and Information

Sciences

$18-4-2005$

\title{
Sliding Mode Control of a Piezoelectric Actuator with Neural Network Compensating Rate-Dependent Hysteresis
}

S. Yu

Dalian Maritime University, China

G. Alici

University of Wollongong, gursel@uow.edu.au

B. Shirinzadeh

Monash University

J. Smith

Monash University

Follow this and additional works at: https://ro.uow.edu.au/engpapers

Part of the Engineering Commons

https://ro.uow.edu.au/engpapers/76

\section{Recommended Citation}

Yu, S.; Alici, G.; Shirinzadeh, B.; and Smith, J.: Sliding Mode Control of a Piezoelectric Actuator with Neural Network Compensating Rate-Dependent Hysteresis 2005.

https://ro.uow.edu.au/engpapers/76

Research Online is the open access institutional repository for the University of Wollongong. For further information contact the UOW Library: research-pubs@uow.edu.au 


\section{Sliding Mode Control of a Piezoelectric Actuator with Neural Network Compensating Rate-Dependent Hysteresis*}

\author{
Shuanghe $\mathrm{Yu}$ \\ Automation Research Center \\ Dalian Maritime University \\ Dalian, China 116026 \\ shuanghe@newmail.dlmu.edu.cn \\ Gursel Alici \\ Faculty of Engineering \\ University of Wollongong \\ NSW 2522, Australia \\ gursel@uow.edu.au
}

\author{
Bijan Shirinzadeh \\ Department of Mechanical Engineering \\ Monash University \\ VIC 3800, Australia \\ bijan.shirinzadeh@eng.monash.edu.au \\ Julian Smith \\ Department of Surgery \\ Monash University \\ VIC 3800, Australia \\ julian.smith@med.monash.edu.au
}

\begin{abstract}
Piezoelectric actuators (PEA) are the fundamental elements for high-precision high-speed positioning/tracking task in many nanotechnology applications. However, the intrinsic hysteresis observed in PEAs has impaired their potential, specially, the motion accuracy. In this paper, the complicated nonlinear dynamics of PEA including hysteresis, creep, drift and time-delay etc. are treated as a black-box system exhibited as rate-dependent hysteresis. The multi-valued hysteresis is analyzed as a single-valued function so that a neural network (NN) can be built to model the hysteresis and its inversion. A sliding mode controller (SMC) augmented with inverse hysteresis model is then developed to compensate the hysteretic behavior, modeling error and disturbance to improve the positioning/tracking stability and accuracy. The effectiveness of this algorithm experimentally verified through the actual tracking control of a PEA.
\end{abstract}

Index Terms - hysteresis, neural network, sliding mode, piezoelectric actuator.

\section{INTRODUCTION}

Due to its rapidly growth and promising innovation in modern industry and research field, nanotechnology has been emerging as one of the most popular technical fields currently being considerably explored ${ }^{[1]}$. One of the pivotal requirements in nanotechnology is nanomanipulation, which is required to deliver nanometer level resolution for handling micro- or nano-scaled objects while maintaining other characteristics such as stiffness, force, speed and adequate dynamic range ${ }^{[2]}$. Due to their nanometer resolution, high stiffness, big driving force and fast response, piezoelectric actuators (PEA) have been recognized as the fundamental elements to actuate the nanomanipulation systems for managing extremely small displacements ${ }^{[3]}$. However, the potential of these actuators has been impaired by the adverse effects of their highly nonlinear input/output behavior, more specifically, the hysteresis inherent to piezoelectric materials between the applied voltage and the displacement output especially for a relatively long range of motion or high-frequency excitation, resulting in limited accuracy in open-loop control system even instability in the closed-loop control system ${ }^{[4]}$. Therefore, one of the critical challenges for high-precision positioning/tracking control is to break through the bottleneck of the complicated hysteretic nonlinearities of PEAs in order to acquire improved performance. A customary way is to build satisfactory model to describe the behavior of PEA and use inverse compensation to approximately linearize its response ${ }^{[5]}$.

Hysteresis has been studied for decades, and various models have been proposed to efficiently capture the hysteretic characteristics ${ }^{[6]}$. Among these models, Preisach model (PM) is the most popular model for its well-defined and reliable experimental identification procedure based on the measurement of first-order reversal curves. Generally, the classical PM intrinsically describes rate-independent hysteresis, which means that the output signal of a system with hysteresis depends not only on the present input value but also on its past amplitudes, especially its past extrema, but not on its rates. The wiping-out and congruency properties are the necessary and sufficient conditions for a system to be correctly described by a classical PM. However, these properties are seldom shown by real hysteretic systems, and significant rate-dependence of the hysteresis loops has been observed in many experiments, i.e., the width increases and the tilt decreases as the driving voltage frequency increases ${ }^{[7-8]}$. The changes are likely due to the cumulative effects of the viscous-type and other dynamic factors, which clearly become more pronounced as the voltage frequency increases. Therefore, the accuracy of the classical PM in modeling PEA behavior is expected to deteriorate as the frequency of the driving voltage varies. In order to overcome these limitations, A few dynamic generalizations of classical PM have been developed by introducing the dependence of Preisach density function (PDF) on the rate of input or the rate of output ${ }^{[9-12]}$. With two consecutive voltage extrema as inputs, the variation rate between the extrema as an additional input, a feedforward

"This work is supported by Australian Research Council (ARC) Discovery and LIEF grants, Monash Research Fund (MRF) and Dalian Maritime University Research Fund. 
neural network (FNN) is developed to address the issue of rate-dependent hysteresis in $\mathrm{PEA}^{[9]}$. Mayergozy's work on the output-rate-dependent PM has been extended to the input-rate-dependent PM with the similar identification and numerical simulation procedure ${ }^{[10]}$. An average-input-ratedependent Preisach-type dynamic model is implemented by experimentally building a database of mirror functions between the arbitrary-frequency excitation and the lowfrequency excitation ${ }^{[11]}$. A rate-dependent Prandtl-Ishlinskii (PI) model, a subclass of PM, is presented to model ratedependent hysteretic characteristic of a PEA by experimentally determining a linear relationship between the slopes of the hysteretic loading curve and the input rate ${ }^{[12]}$. Recently, a two-stage model expressed as an ordinary differential equation with a rate-independent hysteresis and with rate-dependent time-delay is proposed to describe the dynamic hysteresis of a magnetostrictive actuator ${ }^{[13]}$. In addition, PEA also exhibits other undesirable characteristics such as drift and creep ${ }^{[14]}$.

Generally speaking, classical PM can be described as a weighted aggregate effect of all possible hysteresis operators called as hysterons. Based on the geometric similarities between $\mathrm{PM}$ and $\mathrm{NN}$, NNs are being investigated to alternatively implement the characteristics of PM for their universal function approximation ability, where the various standard training algorithms of NN simplify the tedious identification procedure of $\mathrm{PM}^{[15-17]}$. However, same as PM, they essentially implement static hysteresis, i.e., rateindependent hysteresis. In this paper, the complicated nonlinear dynamics of PEA including rate-independent hysteresis, creep, drift and time-delay etc. are treated as a black-box system exhibited as rate-dependent hysteresis, a Feedforward $\mathrm{NN}$ is put forward to describe the hysteresis nonlinearity. Comparing previous models, the proposed model is capable to model rate-dependent hysteresis under different operating condition by introducing input variation rate as additional inputs besides the current input, which change the multi-valued characteristics of hysteresis into a single-valued relation, and relax the requirements on hysteresis to be described by PM. The approximation ability of $\mathrm{NN}$ is investigated using experimental dynamic data inspired by the representative variant-amplitude and variantfrequency sinusoidal input signals and the results confirm that the model can accurately predict the dynamic response of hysteresis nonlinearity in the PEA. Furthermore, the inversion of the rate-dependent hysteresis can be obtained with similar principle in order to compensate its effects. Many efforts have been made to deal with the hysteresis nonlinearity of PEAs for the improved accuracy. Inverse compensation is a fundamental control approach, which has been shown to significantly enhance positioning/tracking performance of PEA. Open-loop feedforward inverse control can be chosen for its simplicity and guaranteed stability. However, the performance of control system is severely susceptible to the modelling accuracy introduced by inverse model and external disturbance. To combat this problem, the closed-loop PID feedback control combining feedforward compensation is used to cope with the effects of residual hysteresis ${ }^{[18-19]}$. In this paper, a robust control approach - sliding mode control augmented with the proposed $\mathrm{NN}$ inverse hysteresis model is designed to compensate the rate-dependent hysteresis, external disturbance and modeling error to achieve desired performance. Experimental results will be provided to show the piezoelectric actuator can precisely track the desired trajectory.

The remainder of the paper is organized as follows. The nonlinear dynamics of PEA is explored in Section II. The rate-dependent hysteresis model, its inversion and the corresponding identification method by $\mathrm{NN}$ are presented in section III. The sliding mode control combined with inverse compensation is developed and the performance is evaluated through experiment of tracking control of a PEA in Section IV. Finally, conclusions are summarised in Section V.

\section{PEA - A SYSTEM WITH HYSTERESIS}

A block diagram shown in Fig. 1 represents the complete model for PEA, which can be expressed as the rate-dependent linear, viscous-type and time-delay etc. dynamics $G_{0}(s)$ cascaded with rate-dependent hysteresis $H$.

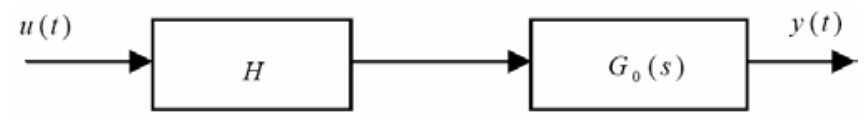

rate-independent hysteresis rate-dependent dynamics

Fig. 1 Model structure of a PEA

The schematic diagram of experimental setup and the block diagram of the PEA system are shown in Fig. 2. The PEA system is comprised of four elements: a piezo actuator with built-in strain gauge providing motion and position feedback, control electronics, data acquisition system and $\mathrm{PC}$ for control and monitoring.

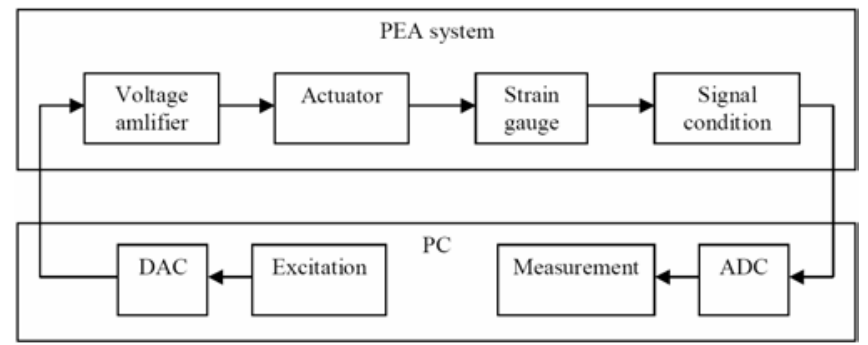

Fig. 2 Block diagram of the open-loop measurement system for a PEA

It is well-known that the width of hysteresis in PEA will increase as the voltage amplitude of excitation signal increases. However, if the voltage amplitude is fixed, the experimental results shown in Fig. 3 indicate that as the voltage frequency of excitation signal increases, the width of the hysteresis curve also significantly increases. If PEA is treated as a black-box, the results indicate that the hysteresis between the input and displacement is rate-dependent.

\section{A FEEDFORWARD NN FOR RATE-DEPENDENT HYSTERESIS}

As has been reported in the past, hysteresis is a major limitation of PEAs. Research efforts have focused on setting up a suitable hysteresis model. Based on the measured firstorder reversal curves under static conditions (e.g. the excitation frequency is low such as $0.1 \mathrm{~Hz}$ ), the classical PM (1) can be used to model rate-independent hysteresis by a parallel summation of a series of elementary hysteresis 
operators, i.e., hysterons. Each hysteron is characterized by a pair of switching values $(\alpha, \beta)$ as shown as Fig. 4.

$$
\begin{aligned}
& y(t)=\iint_{\alpha \geq \beta} \mu(\alpha, \beta) \gamma_{\alpha \beta}(u(t)) d \alpha d \beta \\
& y(t)=\sum_{i=1}^{N} \sum_{j=1}^{N} \mu\left(\alpha_{i}, \beta_{j}\right) \gamma_{\alpha_{i} \beta_{j}}(u(t))
\end{aligned}
$$

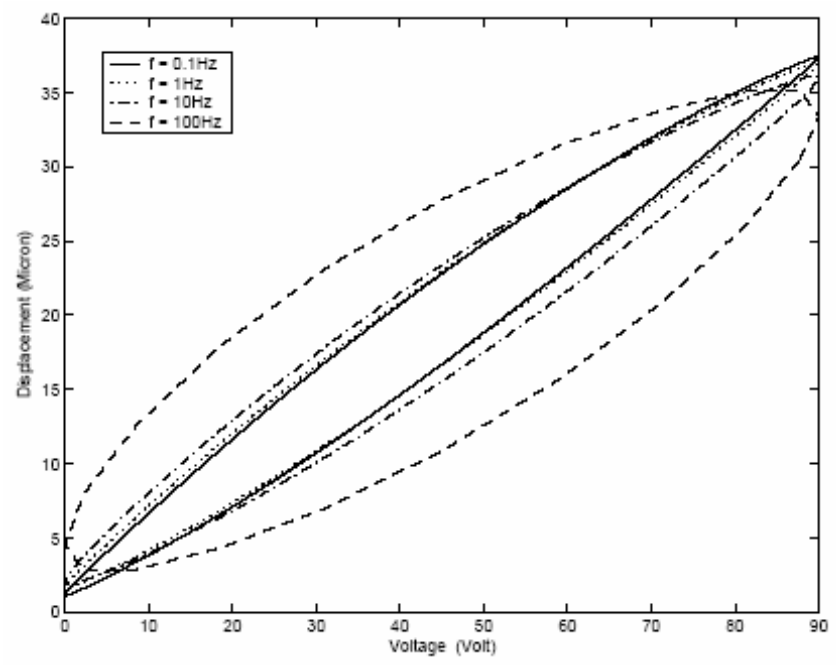

Fig. 3 Hysteresis with fixed-amplitude different-frequency excitation

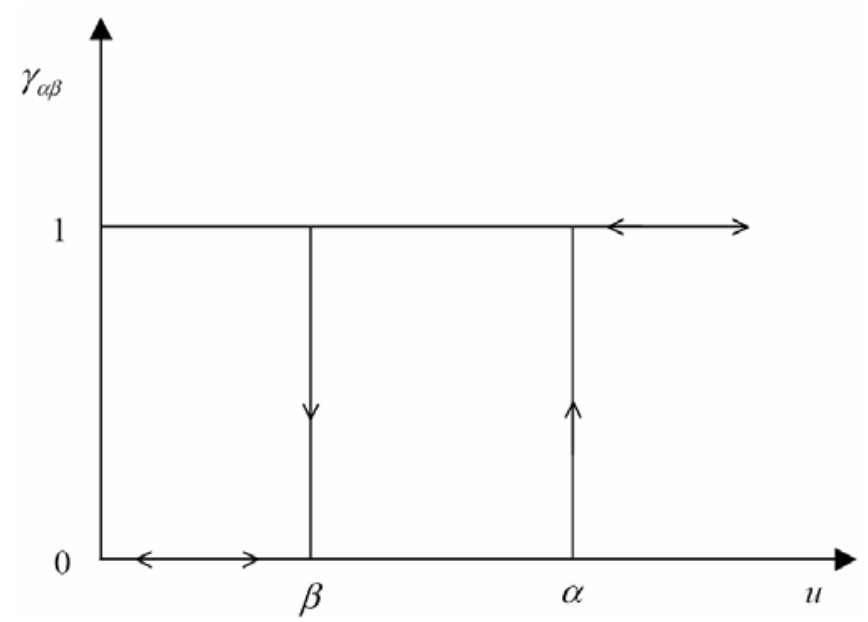

Fig. 4 Elementary hysteresis operators

Due to its intrinsical rate-independent property, the classical PM (1) or (2) is not suitable for modeling PEA behavior across wide frequency band. The dynamic Preisach model (3), taking the Preisach distribution function $\mu$ as a function of the input rate and treat the history of input extremum in the same way as the classical PM, provides very accurate results, but tends to be computationally intensive.

$$
y(t)=\iint_{\alpha \geq \beta} \mu(\alpha, \beta, \dot{u}) \gamma_{\alpha \beta}(u(t)) d \alpha d \beta
$$

The geometric description of the classical PM shown in Fig. 5 can raise some analogies with the definition of feedforward neural networks (FNN). Mathematical models based FNN may form an alternative to classical PM. However, the multi-value mapping characteristics of hysteresis nonlinearity bring about difficulties for FNN to approximate it, since there may be multiple possible outputs for a given input. Therefore, a single-valued mapping must be defined to describe hysteresis nonlinearity.

In this paper, a kind of architecture of FNN is proposed in order to train the experimental data from a PEA to approximate its hysteretic characteristics. The input data of FNN are the current input $u$ and the input rate $\dot{u}$. Thus, the mathematical relation between the current displacement and these two inputs becomes an one-to-one mapping, which guarantees the complex rate-dependent hysteresis can be approximated by the FNN.

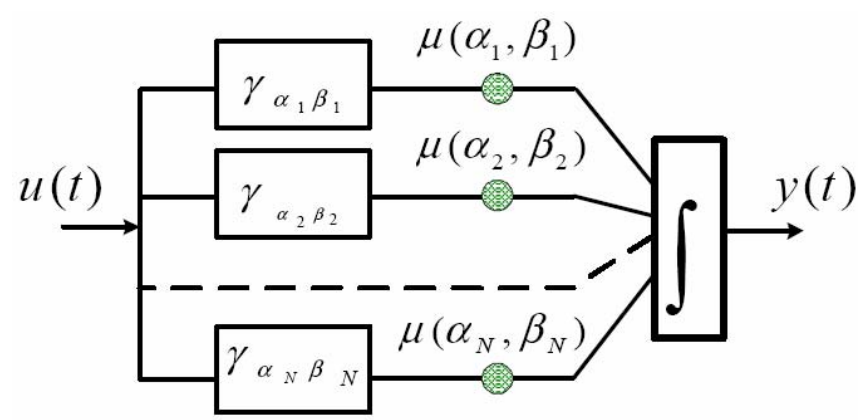

Fig. 5 The geometric description of the classical PM

Fig. 6 shows the hysteretic characteristics of the PEA excited by a fixed-amplitude and decreasing-frequency sinusoidal voltage input signal, and FNN well approximate its behaviour.

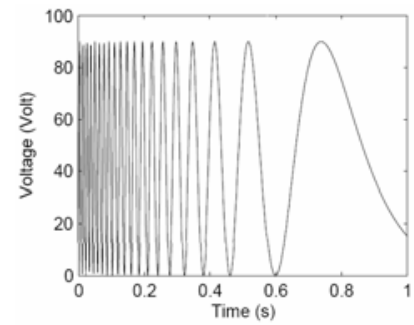

(a) Excitation

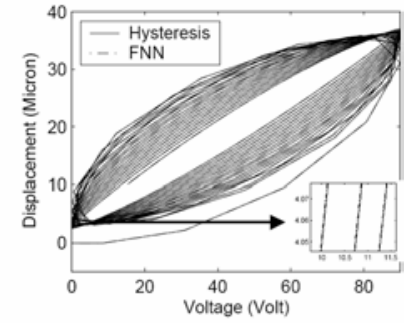

(b) Hysteresis
Fig. 6 Experimental hysteresis and FNN model

For the excitation voltage signal with increasingfrequency and decreasing-magnitude or decreasingfrequency and decreasing-magnitude, similar results shown in Fig. 7 and Fig. 8 are obtained respectively.

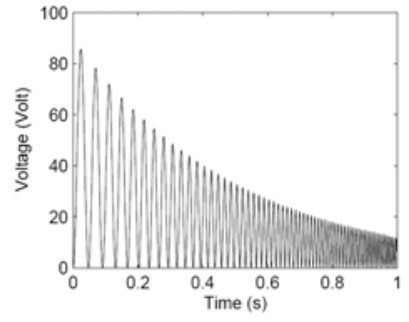

(a) Excitation

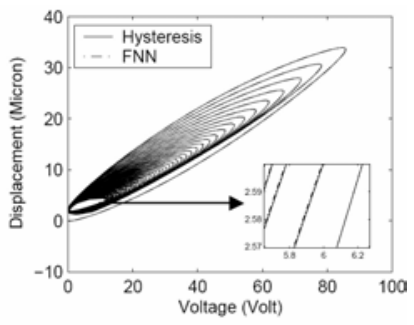

(b) Hysteresis
Fig. 7 Experimental hysteresis and FNN model 


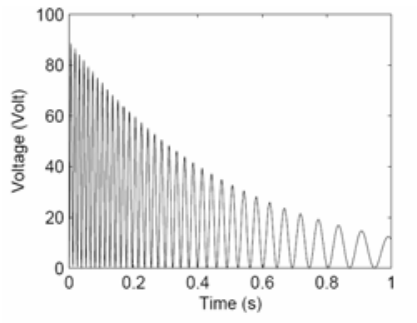

(a) Excitation

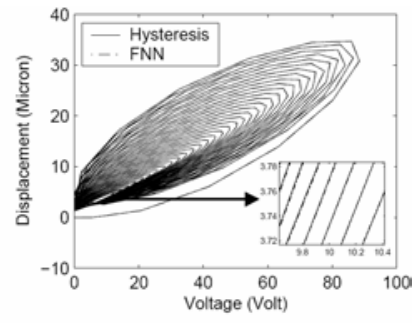

(b) Hysteresis
Fig. 8 Experimental hysteresis and FNN model

It must be noted that the experimental data are collected in the form of a decreasing oscillating sequence in full motion range of amplitude and frequency. Such a sequence would implicitly contain considerable information of the hysteretic characteristics required to be extracted.

\section{SLIDING MODE CONTROL SYSTEM DESIGN}

In this section, a robust control approach - sliding mode control is selected to compensate the bounded modeling error and external disturbance. Firstly, a NN model shown in Fig. 9 is set up for inverse hysteresis based on the similar principle as section III. The obtained inverse model will be augmented with sliding mode control to compensate the effects of hysteresis.

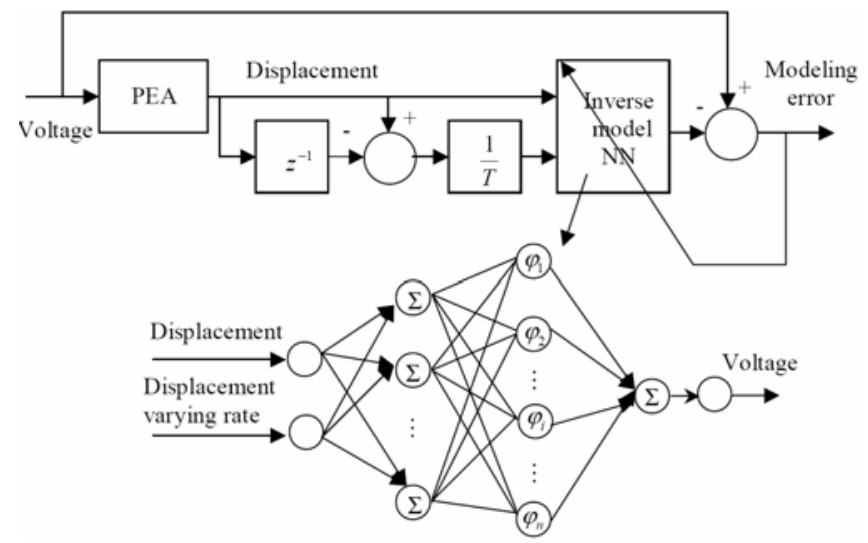

Fig. $9 \mathrm{NN}$ inverse hysteresis model

The first step in the sliding mode control design is to define a sliding surface, which renders the dynamic system stable when the system lies on the sliding surface, i.e., $s=0$. In the case of second order systems, the sliding surface is typically chosen as a first order function

$$
s=\dot{e}+\lambda e
$$

where $\lambda$ is a positive parameter, $e=y-y_{d}$ is tracking error. Therefore in the sliding surface $s=0, e=0$ and $\dot{e}=0$ are asymptotically stable equilibrium. The control objective is to make the sliding dynamics in (4) stable even though the bounded uncertainty and external disturbance. Therefore the control law is chosen as:

$$
u=u_{N N}-\eta s-\rho \frac{s}{|s|+\varepsilon}
$$

the parameters $\eta$ and $\varepsilon$ are positive constants and $\rho$ is chosen to counteract the uncertainties. The last term is a continuous approximation to the discontinuous function used in sliding mode control. $\rho$ is chosen to guarantee that the sliding dynamics are stable and ultimately bounded. Since the continuous approximation is used instead of the discontinuous function for the sliding mode control, the tracking will not be guaranteed to go to zero. However, the tracking error is bounded based on the choice of $\eta$ and $\varepsilon$ and can be determined using Lyapunov stability theory to be $|s| \leq \varepsilon /(\eta-1)$. The bound on the tracking error is then found to be $|e| \leq \varepsilon /(\lambda(\eta-1))$. Therefore the tracking error can be improved by increasing $\eta, \lambda$ and decreasing $\varepsilon$. However, as the tracking error is improved by tuning these parameters, the controller approaches a discontinuous one, which can cause chattering and high frequency oscillations. The experimental results are shown in Fig. 10 and Fig. 11, where rate-dependent hysteresis is approximately linearized, and tracking precision is improved greatly with the inverse rate-dependent hysteresis compensation.

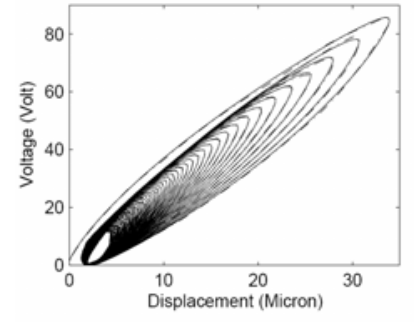

(a) Inverse Hysteresis

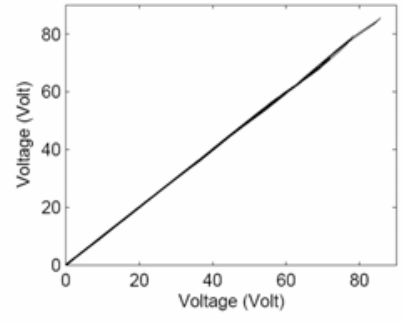

(b) Linearization
Fig. 10 Linearization of the hysteresis in PEA

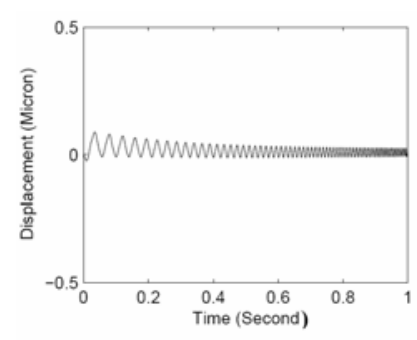

(a) Without compensation

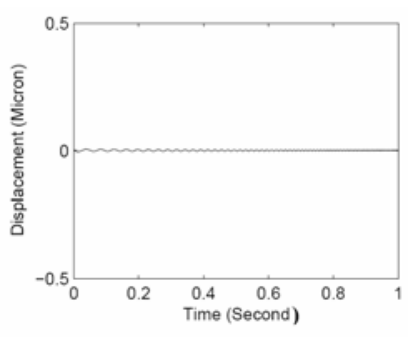

(a) With compensation
Fig. 11 The comparison of tracking performance

\section{CONCLUSIONS}

In this paper, the rate-dependent property of a PEA is completely investigated by a few representative sinusoidal voltage signals, and a kind of FNN is proposed to model the complex hysteretic behaviour successfully. Furthermore, the similar algorithm is extended to model the inversion of ratedependent hysteresis, which is combined with sliding mode control. As a result, the higher-precision tracking ability is obtained. In the next stage of the research, we will test the control algorithm under a load. i.e., while actuating one of our micromanipulation mechanisms. This will attest the robustness of the algorithm to disturbances.

\section{REFERENCES}

[1] K. Spanner and S. Vorndran, "Advances in piezo-nanopositioning technology," IEEE/ASME International Conference on Advanced 
Intelligent Mechatronics, Kobe, Japan, pp. 1338-1343, July 20-24, 2003.

[2] A. G. Requicha, "Nanorobots, NEMS and nanoassembly," Proceedings of the IEEE, vol.91, no. 11, pp. 1922-1933, Nov. 2003.

[3] N. Bonnail, D. Tonneau, F. Jandard, G. A. Capolino and H. Dallaporta, "Variable structure control of a piezoelectric actuator for a scanning tunneling microscope," IEEE Transactions on Industrial Electronics, vol. 51, no. 2, pp. 354-363, April 2004.

[4] H. Adriaens, W. L. De Koning and R. Banning, "Modeling piezoelectric actuators," IEEE/ASME Transactions on Mechatronics, vol. 5, no. 4, pp. 331-341, Dec. 2000.

[5] J. J. Tzen, S. L. Jeng and W. H. Chieng, "Modeling of piezoelectric actuator for compensation and controller design," Precision Engineering, vol. 27, no. 1, pp. 70-86, Jan. 2003.

[6] I. D. Mayergoyz, Mathematical Models of Hysteresis, New York, Springer-Verlag, 1991.

[7] P. Ge and M. Jouaneh, "Modeling hysteresis in piezoceramic actuators," Precision Engineering, vol. 17, pp. 211-221, 1995.

[8] P. Ge and M. Jouaneh, "Generalized Preisach model for hysteresis nonlinearity of piezoceramic actuators," Precision Engineering, vol. 20, pp. 99-111, 1997.

[9] D. Song and C. J. Li, "Modeling of piezo actuator's nonlinear and frequency dependent dynamics," Mechatronics, vol. 9, pp. 391-410, 1999.

[10]Y. Yu, Z. Xiao, N. G. Naganathan and R. V. Dukkipati, "Dynamic Preisach modelling of hysteresis for the piezoceramic actuator system," Mechanism and Machine Theory, vol. 37, no. 1, pp. 75-89, Jan. 2002.

[11]R. B. Mrad and H. Hu, "A model for voltage-to-displacement dynamics in piezoceramic actuators subject to dynamic-voltage excitations," IEEE/ASME Transactions on Mechatronics, vol. 7, no. 4, pp. 479-489, Dec. 2002.

[12]W. Ang, C. Riviere and P. Khosla, "Modeling Rate-dependent Hysteresis in Piezoelectric Actuator," Proceedings of the 2003 IEEE/RSJ International Conference on Intelligent Robots and Systems, pp. 1975-1980, 2003.

[13]D. Davino, C. Natale, S. Pirozzi and C. Visone, "Phenomenological dynamic model of a magnetostrictive actuator," Physica B: Condensed Matter, vol. 343, no. 1-4, pp. 112-116, 2004.

[14]H. Richter, E. A. Misawa, D. A. Lucca and H. Lu, "Modeling nonlinear behavior in a piezoelectric actuator," Precision Engineering, vol 25, no. 2, pp. 128-137, 2001.

[15]A.A. Adly and S.K. Abd-Hafiz, "Using neural networks in the identification of Preisach-type hysteresis models," IEEE Transactions on Magnetics, vol. 34, no. 3, pp. 629-635, 1998.

[16]M. Kuczmann and A. Ivanyi, "A new neural-network-based scalar hysteresis model," IEEE Transactions on Magnetics, vol. 38, no. 2, pp. 857-860, 2002.

[17]C. Li and Y. Tan, "A neural networks model for hysteresis nonlinearity," Sensors and Actuators A: Physical, vol. 112, no. 1, pp. 49-54, 2004.

[18]P. Ge and M. Jouaneh, "Tracking Control of a Piezoceramic Actuator," IEEE Transactions on Control System Technology, vol.4, no.3, pp. 209-216, May 1996.

[19]M. Goldfarb and N. Celanovic, "Modeling Piezoelectric Stack Actuators for nControl of Micromanipulation," IEEE Control System Magazine, vol.17, no. 3, pp. 69-79, June 1997. 